\title{
Chemical reactions in supercritical carbon dioxide
}

\author{
Zou Gang, Jiang Huan-Feng*, Chen Ming-Cai \\ Guangzhou Institute of Chemistry, Chinese Academy of Sciences, 510650 Guangzhou \\ E-mail: jhf@mail.gic.ac.cn
}

\section{Dedicated to Professor Zhi-Tang Huang on his $75^{\text {th }}$ birthday}

(received 11 Feb 03; accepted 30 June 03; published on the web 05 July 03)

\begin{abstract}
This review presents recent advances of chemical reactions in supercritical carbon dioxide in our group. The reactions include carbonylation, oxidation, radical reaction, polymerization, etc.
\end{abstract}

Keywords: Supercritical carbon dioxide, green chemistry, reaction

\section{Introduction}

Resources and the environment are the two major issues that mankind pays close attention to. With the unceasing expansion of population, the utilizable resources on the earth are becoming less and less. At the same time, because people cannot put resources to reasonable use, a large amount of resources return to nature in the shape of waste gas and residue, seriously polluting the earth that man relies on. How to solve the problem of resources and the environment is of vital importance to mankind's existence and development. Formed in the 1990s, the global environmental-development strategy connects the settlement of environmental problems with the optimum process of resources use. As to chemistry, the purpose is to ensure that chemical reactions, raw materials, catalysts, media and products are all environmentally friendly by stopping the pollution at source.

As a reaction medium the attractive physical and toxicological inertness properties of supercritical carbon dioxide $\left(\mathrm{scCO}_{2}\right)^{[1]}$ have made it superior to conventional organic solvents. First, $\mathrm{scCO}_{2}$ has liquid-like density and gas-like viscosity and consequently leads to high solubility and rapid mass transfer velocity. Secondly, a slight change of pressure, especially near the critical point of $\mathrm{CO}_{2}$, enable the solvent properties such as density, viscosity, diffusivity and polarity to change constantly from approximate gaseous to similar liquid state. Thirdly, separating $\mathrm{CO}_{2}$ from the reaction mixture is energy-efficient and a product can be obtained by simple treatment. More importantly, $\mathrm{CO}_{2}$ is inexpensive, nonflammable, nontoxic, environmentally friendly and one of the reaction media, which is favored for Green chemistry research.

At present, $\mathrm{scCO}_{2}$ has been using as a medium in the following chemical reactions: enzyme-catalyzed reactions ${ }^{[2]}$, polymerizations ${ }^{[3]}$, radical reactions ${ }^{[4]}$, cycloaddition reactions ${ }^{[5]}$, transition metal catalyzed reactions and so on. Palladium-catalyzed reactions performed are the Heck 
${ }^{[7]}$ and Stille reaction ${ }^{[8]}$.

\section{Carbonylation}

Carbonylation is a classification of reactions for introducing the carbonyl group into organic molecules. These may be classified according to the particular reaction, thus hydroformylation, hydrocarboxylation and hydroesterification.

\subsection{Alkenes}

The functionalisation of alkenes is one of the most important areas of chemistry. Efficiency of functionalisation increases by a few by tens of times, with high atom efficiency, and there are many examples of clean processes in this area. This allows the development of concentrated, environmentally friendly reactions and technical processes. Conversion and regioselectivity is always an important question for Pd-catalyzed carbonylation. Among many means to raise the reactant conversion and product regioselectivity, the choice of a suitable solvent is one of the most important. When norbornene $\mathbf{1}$ was carbonylated in the presence of $\mathrm{MeOH}$ in $\mathrm{scCO}_{2}$, three products, cis-exo-diester $\mathbf{2}$, cis-exo- $\beta$-chloride ester $\mathbf{3}$ and exo-chloronorbornane $\mathbf{4}$ were obtained. (Scheme 1$)^{[10]}$ For Scheme 1 see page 208.

We found that by adding just the right amount of a polar-solvent improved the dissolution of catalysts in $\mathrm{scCO}_{2}$, enhanced reaction efficiency, and improved the selectivity. The context of "Green Chemistry" confirmed our investigations, it said the utilization of cosolvent promoted the reaction efficiency significantly, and allowed the direct, one-step synthesis of acetate in a highly efficient manner.

The presence of base can increase the selectivity to main product diester $\mathbf{2}$. Different bases had quite different effects, the best result was observed in the reaction using $\mathrm{Et}_{3} \mathrm{~N}$. When 2 equivalents of $\mathrm{Et}_{3} \mathrm{~N}$ was added, the yields of product $\mathbf{2}$ is $82.2 \%$ and that of product $\mathbf{4}$ is $3.4 \%$, the selectivity to product $\mathbf{2}$ reached $96 \%$, meanwhile no product 4 was detected. The selectivity to the major product has been improved remarkably in comparison with the results studied by James and Stille. Our investigation also showed that both high temperature and $\mathrm{CO}_{2}$ pressure have unfavorable effect on the selectivity to product 2. For example, when temperature was raised to $85 \mathrm{C}$, product $\mathbf{4}$ became the major product. Under supercritical conditions compound 5 behaves in the same way (Scheme 2). ${ }^{[12]}$ For Scheme 2 see page 208.

Radical addition reactions provide good examples of the successful use of supercritical carbon dioxide as a reaction medium, as they involve neither nucleophiles nor electrophile and may be carried out in non-polar solvents. ${ }^{[13-14]}$. In $\mathrm{scO}_{2}$, we smoothly realized the Pd-catalyzed radical addition reaction of $\mathrm{CO}$ and $\mathrm{CCl}_{4}$ to 1-octene. ${ }^{[15-16]}$ (Scheme 3). For Scheme $\mathbf{3}$ see page 208.

Under the same catalyst system, comparing the reaction results in a normal organic medium $(\mathrm{EtOH})$ with that in $\mathrm{scCO}_{2}$, we found product $\mathbf{9}$ and product $\mathbf{1 0}$ have quite different selectivities. The amount of carbonylation product is much higher in $\mathrm{scCO}_{2}$. It is likely in supercritical carbon dioxide that $\mathrm{CO}$ has higher solubility and hence carbonylation is favored.

In $\mathrm{scCO}_{2}$, the reaction temperature, the reaction pressure and the amount of alcohol and pyridine all obviously influence the reaction selectivity and conversion. The optimized reaction conditions 
were 1-octene $(2 \mathrm{mmol}), \mathrm{CCl}_{4}$ and $\mathrm{EtOH}(1 \mathrm{~mL})$, pyridine $(4 \mathrm{mmol})$ and $\mathrm{Pd}(\mathrm{OAc})_{2}(0.02 \mathrm{mmol})$ at $60^{\circ} \mathrm{C}$ under $10 \mathrm{MP}$ atm of $\mathrm{CO}_{2}$ for $24 \mathrm{~h}$. When $\mathrm{MeOH}$ or i-PrOH, respectively, was used as substrate both reactions gave satisfactory results.

\subsection{Alkynes}

When examining the Pd-catalyzed carbonylation of alkynes, we found there was competition between carbonylation and dimerziation. ${ }^{[17]}$ Adding alcohol is beneficial to carbonylation up to a certain limit, beyond which both carbonylation and dimerization have lower rates of reaction. This provides the biggest distinction between the common organic solvent and $\mathrm{scCO}_{2}$ (Scheme 4). For

\section{Scheme 4 see page 209.}

In order to explore further the carbonylation of alkynes under common conditions, we put an emphasis on studying the influence of different organic media on carbonylation. The aim was to provide comparable conditions and a theoretical basis for comparing reactions under supercritical reaction conditions, the result is that media affect the stereoselectivity of reactions as well as controlling the chemoselectivity of reactions. ${ }^{[18-22]}$

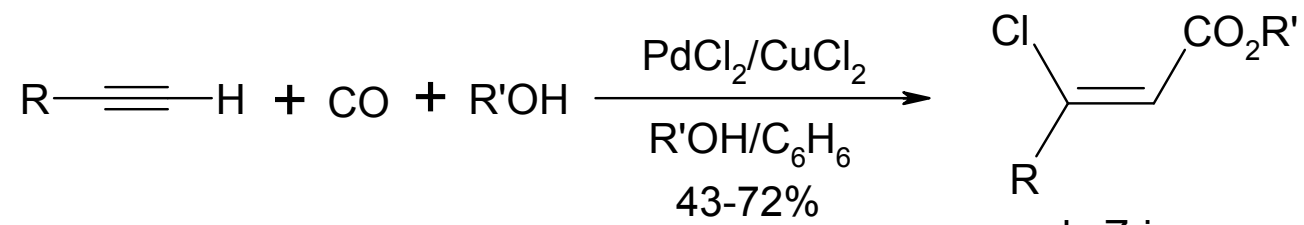

11

only Z-isomers

13

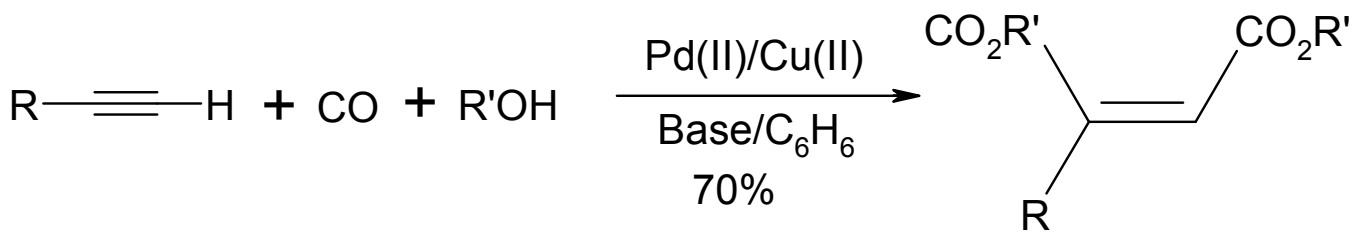

only Z-isomers

11

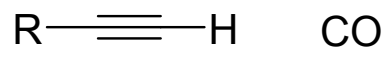

11
14

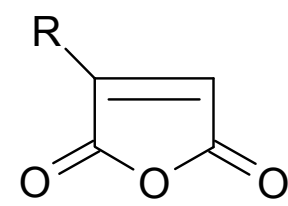

15

\subsection{Amines}

While studying the carbonylation of alkenes, we added a specific amount of various amines as base. When the reaction mixture was analysed, a maximum of carbamate was detected. We thought that it might result from the carbonylation of amines. Carbamate compounds, which have wide and general bioactivity, have been applied as pesticide, germicide, animal-anesthetic and so on. Carbonylation is one of the prospective methods for synthesizing such compounds. 
On the basis of certain results we got under normal conditions, we investigated the reaction under supercritical conditions ${ }^{[23-24]}$. The chemoselectivity of amine carbonylation in $\mathrm{scCO}_{2}$ and in common organic solvents are not alike. In $\mathrm{MeOH}$, only carbamate was obtained in the presence of $\mathrm{O}_{2}$ while in $\mathrm{MeOH} / \mathrm{sCCO}_{2}$, methyl $\mathrm{N}$-n-butylcarbamate and oxalbutyline were obtained and the ratio of methyl $\mathrm{N}$-n-butylcarbamate to oxalbutyline could be regulated with the amount of $\mathrm{MeOH}$. In the absence of $\mathrm{O}_{2}$, oxalbutyline was obtained primarily.

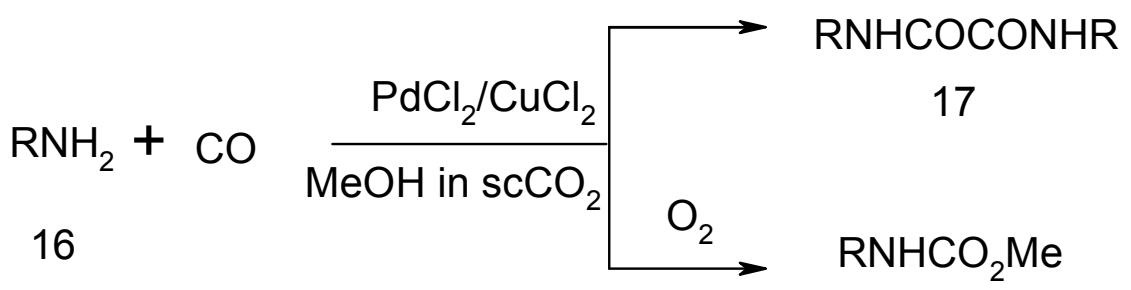

18

\section{Oxidation}

When studying the carbonylation of 1-octene, the reaction gave not the anticipated carbonylation products but Wacker reaction products. So, we probed the reaction rules for different olefinic oxidation in $\mathrm{scCO}_{2}$ and obtained some interesting results.

\subsection{Wacker reaction}

Wacker reaction is a typical example for applying transition metal catalysts in industry successfully. By the one-step synthetic method, it oxidize alkene to aldehyde(ketone). The following is the process: $\mathrm{Pd}^{2+}$ is reduction to $\mathrm{Pd}^{0}$ while oxidizing alkenes to aldehyde(ketone), then $\mathrm{CuCl}_{2}$ oxidize $\mathrm{Pd}^{0}$ to $\mathrm{Pd}^{2+}$ again, at last $\mathrm{O}_{2}$ oxidize $\mathrm{Cu}^{+}$to generated $\mathrm{Cu}^{2+}$. So the net reaction resulting only includes transformation of alkene and consumption of $\mathrm{O}_{2}$. Lloyd and Luberoff ${ }^{[25]}$ reported that the oxidation reaction of 1-octene(8) in n-propanol gave 2-octanone(19), 3-octanone (20) and 4-octanone (21) in $68 \%, 9 \%$, 3\% yield respectively.

We compared the nature of the products of the Wacker reaction of 1-octene in pure $\mathrm{scCO}_{2}$, pure methanol or low carbon alcohol- $\mathrm{sCCO}_{2}$ (Equation 5$)^{[26]}$. If the reaction time is kept the same, the experiments showed that compared with $\mathrm{MeOH}$, the oxidation of 1-octene in $\mathrm{scCO}_{2}$ has higher selectivity to the major product methyl ketone 19, isomeric ketone $\mathbf{2 0}$ and $\mathbf{2 1}$ were formed only in very low yields. However, the reaction rate is slower, this is because salts such as $\mathrm{PdCl}_{2}$ and $\mathrm{CuCl}_{2}$ lack solubility in $\mathrm{scCO}_{2}$, so the reaction system is heterogeneous.

\section{For Scheme 5 see page 209.}

In low carbon alchol-scCO 2 , since $\mathrm{PdCl}_{2}$ and $\mathrm{CuCl}_{2}$ both dissolve in methanol, which is miscible with $\mathrm{scCO}_{2}$, partial catalysts can dissolve in $\mathrm{scCO}_{2}$. The experimental results showed that the rate of reaction was strikingly increased similar to what was found in methanol alone, with the selectivity towards methyl ketone 19 raised notably (85.5\%). The main by-product was acetal (22)

Raising the reaction temperature and pressure led to acceleration of the reaction rate and increase in the yield of $\mathbf{1 9}$.

By comparing the by-products of reaction under different conditions, some conclusions can be 
drawn: when low carbon alcohol (e.g. $\mathrm{MeOH}$ ) was used as reaction medium, the main by-products were 3-octanone and 4-octanone. Using $\mathrm{scCO}_{2}$ as the medium, the by-products were still 3-octanone and 4-octanone, but their amounts were decreased greatly when $\mathrm{ROH}-\mathrm{scCO}_{2}$ was used as the reaction medium, the main by-product was acetal. It was found that 3-octanone and 4-octanone are produced from inner octenes, such as 2-, 3- and 4-octene. This result can be attributed to isomerization of 1-octene. The acetal was obtained via oxidation of the terminal carbon in 1-octene by the oxygen nucleophile.

We obtained some information by using $\mathrm{scCO}_{2}$ or $\mathrm{ROH}-\mathrm{scCO}_{2}$ as reaction media, namely $\mathrm{scCO}_{2}$ may affect the regioselectivity of olefinic oxidation, and reaction rules could be obtained for alkenes with different electronegativity. So we studied the Wacker reaction of styrene and cyclohexene. ${ }^{[26]}$

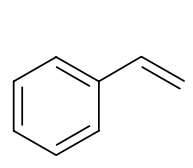

23

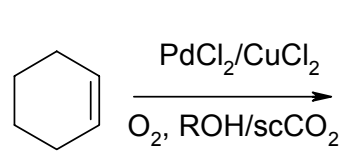

26

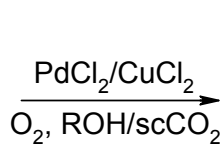$$
\mathrm{CO}_{2}
$$

$\sqrt{20}$

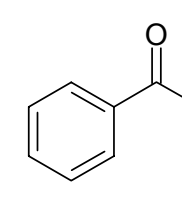

24

$3.8-17.4 \%$

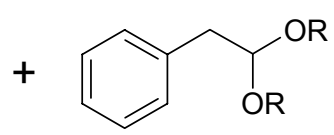

25

$6.5-28.0 \%$

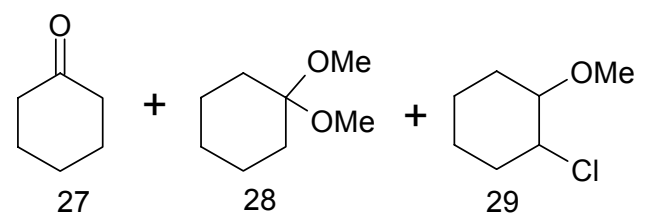

$17.5 \%$

We observed an interesting phenomenum: in normal organic solvents: the Wacker process for styrene and cyclohexene proceeded very smoothly, both gave the expected products acetophenone(24) and cyclohexanone(27), but in $\mathrm{scCO}_{2}$ a considerable amount of acetal was formed. To some extent it revealed some specific characteristics of $\mathrm{scCO}_{2}$, it also revealed the influence of electronegativity or substituting group to oxidation regioselectivity.

So we then selected alkenes with electrophilic groups to study the oxidation, the aim is for acetal outcomes.

\subsection{Acetalization reaction}

Alkyls 3,3-dialkoxypropanoates are important intermediates in organic synthesis and have been used to synthesize a variety of compounds, including coumarins, prophyrins, spermine metabolites and loganin. The synthesis of alkyl 3,3-dialkoxypropanoates is often carried out using transition metal catalysts in DME. However, high yields of alkyl 3,3-dialkoxypropanoates (92\%) were afforded only in the presence of HMPA. In the absence of HMPA the yield was only 46\%. HMPA, a phosphatic organic solvent, is very toxic and harmful to people. How to find the substitute for such solvent has been attracting people's interest.

In $\mathrm{scCO}_{2}$ we close our work on oxidation of methyl acrylate bearing electron-withdrawing groups. Our results show that $\mathrm{Pd}^{2+} /\left(\mathrm{Cu}^{+}\right.$or $\left.\mathrm{Cu}^{2+}\right)$-catalyzed oxidation of methyl acrylate (30) could be successfully processed to give the dimethyl acetal as major product in high conversion (99.4\%) and yield (96.6\%). No ketone was formed (SCheme 6) ${ }^{[27]}$ For $\underline{\text { scheme } 6}$ see page 209. 
In conclusion, from the reactions discussed it can be said that (1) $\mathrm{scCO}_{2}$ is a useful probe for studying both the electronegativity of alkenes and their substituting group and as a reaction medium that has a great effect on the regioselectivity of oxidation reactions. (2) It can be used as a replacement for toxic solvents to offer an environmentally friendly medium for the development of new methods for green synthesis.

In a similar way, we synthesized 3,3-dimethoxyl propylonitrile (35) (Scheme 7) ${ }^{[28]}$ For Scheme 7 see page 210.

\subsection{Etherification reaction}

We studied oxidation using alkyl ethers as the substrates, the reactions both gave satisfactory results when $\mathrm{CO}_{2}$ is in supercritical condition or in liquid condition. (Scheme 8). For Scheme 8 see page 210.

This reaction, with mild reaction condition and high selectivity, is suitable for protecting not only primary alcohols but also secondary and teritiary alcohols. The additive reactions of secondary alcohol and primary alcohol are quicker, on the whole can react completely within $12 \mathrm{~h}$. Addition products of allyl alcohol, propargyl alcohol and chloropropanol are formed in yields of 93\%, 93\%, 99\% and that of isopropyl alcohol in 97\% yield. Although benzoic alcohol, cinnamyl alcohol is also primary alcohol, they have slower addition rate, their conversions are 82\%and 66\% respectively after 12h. The conversion of tertiary butanol is only 50\%, perhaps as the result of solubility or steric inhibition. Extending reaction time can promote their conversion. Conversion of tertiary butanol and cinnamyl alcohol reached $85 \%$ and $92 \%$ separately.

\section{Dimerization and Trimerization}

When investigating the carbonylation using $\mathrm{scCO}_{2}$ as a medium, we found competition between olefinic carbonylation and coupling reaction. ${ }^{[17]}$ In order to clarify the coupled reaction under $\mathrm{scCO}_{2}$ condition (Equation 9) we selected Glaser Coupling as object of study (Scheme 9). Our investigation showed in the presence of amines, reaction was complex and gave low conversion while the addition of $\mathrm{NaOAc}$ plays an important role in enhancing the reaction yield and conversion, this reaction is more environmentally friendly and shows potential utility in industry. For Scheme $\mathbf{9}$ see page 210.

Trimerization is one important method for synthesizing benzene derivatives. We found cupric chloride not only can promote the rate of trimerization but also is a necessary factor for the trimerization.of dissymmetric alkenes (Scheme 10$)^{[31]}$ For Scheme 10 see page 210.

\section{Copolymerization}

Water-soluble polymers are of great importance to both academic study and practical application. ${ }^{32-33}$ Modified with hydrophobic groups, their rheological features will improve greatly. These so-called hydrophobically modified water-soluble polymers are usually produced by copolymerization of water-soluble polymer monomer and hydrophobes. Owing to the intermolecular hydrophobic association, they exhibit unique properties in solution such as greatly enhanced viscosity and its large 
shear dependence, which enable such polymers to have wide application in water treatment, food, cosmetics, personal care products, etc.

Copolymerization of FOA, FUA with acrylic acid can produce hydrophobically modified ploy. Traditionally, these kinds of copolymers are synthesized in organic solvents such as benzene, dioxane. Now we have successfully carried out the copolymerization in supercritical $\mathrm{CO}_{2}$, the polymers were obtained in the form of a dry white powder with diameter of about $0.2 \mu \mathrm{m}$. The properties of such copolymers were fully studied. For example, when FUA content in copolymer reaches $1.5 \mathrm{~mol} \%$, the viscosity of the $2 \%$ aqueous solution of the copolymer is 500 times higher than that of poly (acrylic acid). In addition, the copolymer solutions show strong thixotropy, i.e. under constant shear rates, its viscosity decrease sharply; when the shear is stopped the viscosity recovers again.
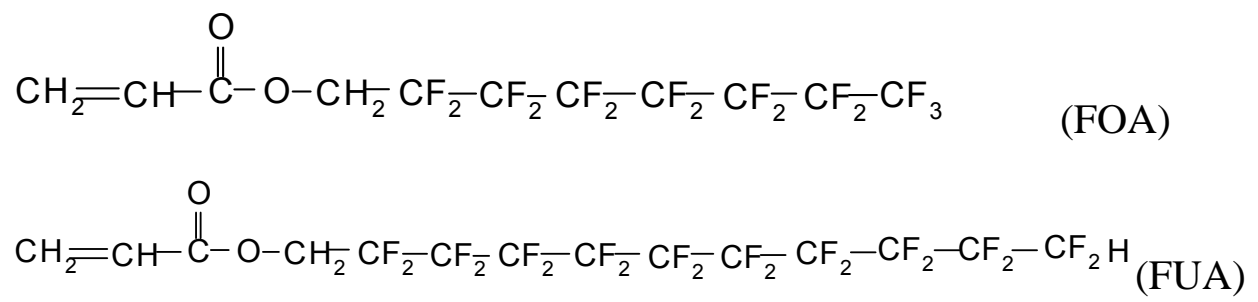

Then we used siloxane-containing monomer replacing the fluorine-containing monomer. The copolymerization of TMSPMA with acrylic acid was also successfully carried out in $\mathrm{scCO}_{2}$. The products were obtained as a dry white powder that contained particles with a diameter of about $0.2 \mu$ $\mathrm{ml}$. It was found that the rheological properties of TMSPMA-modified polyacrylic acid are similar to that of FUA and FOA modified polyacrylic acid. From the point of view of economy, siloxane-containing monomers are much cheaper than fluorine-containing ones, so this work may have meaningful in application.

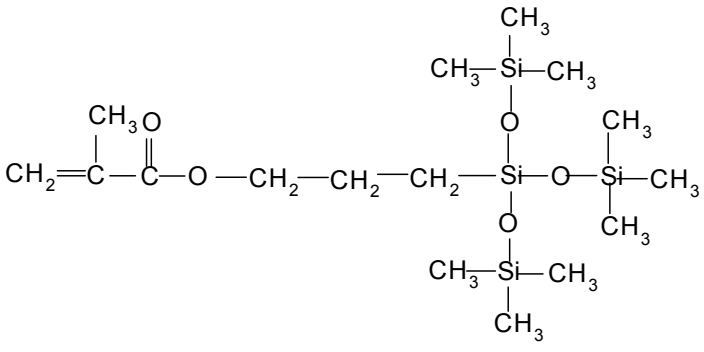

( TMSPMA)

\section{Conclusions}

From the above investigations, the following conclusions may be drawn.

(1) The condition (temperature, pressure) of the supercritical fluid can affect the reaction conversion and selectivity remarkably.

(2) Owing to the high diffusion and low viscosity of supercritical fluid, collision of reactant molecules and separation of resultant molecules from interfaces are much easier. Consequently, the results gave higher conversion and selectivity than under conventional organic solution condition.

(3) The addition of a small amount of co-solvents regulates the ratio of products and thus achieves the 
goal of raising selectivity.

(4) The supercritical $\mathrm{CO}_{2}$ fluid can reduce the use of toxic substance.

\section{References}

1. (a) Jessop, P. G.; Leitner, W. "Chemical Synthesis Using Supercritical Fluids”, Wiley-VCH, Weinheim, 1999, p1. (b) Li, J.; Jia, L.; Jiang, H. Chin. J. Org. Chem. 2000, 20, 293.

2. Randolph, T. W.; Clark, D. S.; Blanch, H. W.; Prausnitz, J. M. Science, 1988, 239, 387.

3. Desimone, J. M.; Guan, Z. S. Science, 1992, 257, 945.

4. Hadida, S.; Super, M. S.; Beckman, E. J.; Curran, D. P. J. Am. Chem. Soc. 1997, 119, 7406.

5. Lsaacs, N. S.; Keating, N. J. Chem. Soc., Chem. Commun. 1992, 876.

6. Burk, M. J.; Feng, S. G.; Gross, M. F.; Tumas, W. J. Am. Chem. Soc. 1995, 117, 8277.

7. Carroll, M. A.; Holmes, A. B. Chem. Commun. 1998, 1395.

8. Morita, D. K.; Pesiri, D. R.; David, S. A.; Glaze, W. H.; Tumas, W. Chem. Commun. 1998, 1397.

9. Li, J.; Jiang, H. Huaxue Tongbao 2000, 13.

10. Jia, L.; Jiang, H.; Li, J. Green Chem. 1999, 1, 91.

11. James, D. E.; Stille, J. K. J. Am. Chem. Soc. 1976, 98, 1810.

12. Jia, L.; Jiang, H.; Li, J.; Sun, J.; Tan, X.; Feng, A.; Li, G. Journal of Xiamen University (Natural Sciences) 1999, 38(Sup.), 246.

13. Jasperse, C. P.; Curran, D. P.; Fevig, T. L. Chem. Rev. 1991, 91, 1237.

14. Motherwell, W.; Crich, D. Free Radical Chain Reaction in Organic Synthesis, Academic Press, London.

15. Sun, J.; Jiang, H. Chin. J. Chem. 2001, 19, 518.

16. Sun, J.; Jiang, H. Journal of Lanzhou University (Natural Sciences) 2000, 36, 75.

17. Li, J.; Jiang, H.; Li, G. Guangzhou Chemistry 2000, 1.

18. Li, J.; Jiang, H.; Feng, A.; Jia, L. J. Org. Chem. 1999, 64, 5984.

19. Li, J.; Jiang, H.; Jia, L. Synth. Commun, 1999, 29, 3733.

20. Li, J.; Jiang, H.; Chen, M. Synth. Commun. 2001, 31, 199.

21. Li, J.; Jiang, H.; Chen, M. Synth. Commun. 2001, 31, 3131.

22. Li, J.; Jiang, H.; Chen, M. Chin. J. Chem. 2001, 19, 1153.

23. Jia, L.; Jiang, H.; Li, J. Guangzhou Chemistry 1999, 32.

24. Li, J.; Jiang, H.; Chen, M. Green Chem. 2001, 3, 137.

25. Lloyd, W. G.; Luberoff, B. J. J. Org. Chem. 1969, 34, 3949.

26. Jiang, H.; Jia, L.; Li, J. Green Chem. 2000, 2, 161.

27. Jia, L.; Jiang, H.; Li, J. Chem. Comm. 1999, 985.

28. Jiang, H.; Jia, L.; Feng, A.; Tan, X. ZL98113345.2, 1998.

29. Jia, L.; Jiang, H.; Li, J.; Feng, A. Huaxue Tongbao 2000, 48.

30. Li, J.; Jiang, H. Chem. Comm. 1999, 2369.

31. Li, J.; Jiang, H. Chen M, J. Org. Chem. 2001, 66, 3627.

32. Hu, H.; He, T.; Feng, J.; Chen, M.; Cheng, R. Polymer 2002, 43, 6357.

33. Hu, H.; Chen, M.; Cheng, R. Polymer 2003, 44, 341. 